\title{
Pragmatic Interests and Imprecise Belief ${ }^{1}$
}

\author{
Brad Armendt \\ School of Life Sciences, \\ Arizona State University, \\ Box 874501, Tempe, AZ 85287-4501, USA \\ armendt@asu.edu
}

Does the strength of a particular belief depend upon the significance we attach to it? Do we move from one context to another, remaining in the same doxastic state concerning $p$, yet holding a stronger belief that $p$ in one context than in the other? For that to be so, a doxastic state must have a certain sort of context-sensitive complexity. So the question is about the nature of belief states, as we understand them, or as we think a theory should model them. I explore the idea, and how it relates to work on imprecise probabilities and second-order confidence.

\section{Introduction: A Little Story.}

You are in a conversation with a student, who asks you: in which part of his paper on truth and probability did Ramsey give that example about the unwholesome yellow toadstools? After a moment, you reply: in the last section of the paper, on the 'logic of truth.' Soon after the student leaves your office, the phone rings. A local radio station invites you to play their quiz game. The prize for a correct answer to their question is valuable - a fabulous overseas vacation, let us say-and they offer you several subjects to choose among. You pick 'Probabilism', and amazingly enough, they ask the very same question. Now you think a little longer; it seems like it was the last section of the paper, but could it have been earlier when Ramsey was talking about beliefs and frequencies?

What, if anything, might be different for you in the two situations? What, if anything, is different about your belief that the yellow toadstool example is in the last section of the paper? This story is not perfect ${ }^{2}$, but it is intended to put us in the neighborhood of our

\footnotetext{
${ }^{1}$ Presented at PSA 2012, San Diego. Thanks to audiences at the EpiConFor Workshop in Nancy, and at the Center for Philosophy of Science in Pittsburgh for discussion of earlier versions of this paper. Particular thanks to Rich Thomason, Mark Wilson, Teddy Seidenfeld, Jim Joyce, Darrell Rowbottom, Bob Batterman, and Paul Teller for helpful conversations about topics raised here.

${ }^{2}$ Since there is little cost to any answer you give, we may think that what you say only loosely indicates how strongly you believe. A better example might have the station allow you to bid for a chance to answer the question. But there are still confounding issues, so let us move on.
} 
subject: the extent to which our beliefs, and how strongly we hold them, depend upon what we take to be at stake on them. Does the strength of a particular belief depend upon the significance we attach to it? Might we move from one context to another, remaining in the same doxastic state concerning $p$, yet holding a stronger belief that $p$ in one context than in the other? In order for that to happen, a belief state must have a certain sort of complexity, a context-sensitivity that, in the presence of one set of stakes, provides a belief of one strength, and in the presence of different stakes, a different strength. So the question being asked is about the nature of belief states, as we understand them, or as a theory should model them.

Are belief states stake-sensitive, or not? The question needs further clarification; I have raised it elsewhere (Armendt 2010), and here the clarification will be brief. Then, after noticing that treatments of stake-sensitive beliefs states are uncommon, I will turn to treatments of imprecise belief and look for connections between imprecision and stakesensitivity. Clarification first.

\section{Stake-sensitivity. ${ }^{3}$}

Think of variations in one's dispositional doxastic states, and not just in which doxastic commitments one happens to entertain on different occasions. One way that our doxastic commitments vary from one occasion to another is by change in the light of new information, or newly appreciated information. So learning, or belief change driven by new evidence, even if through stable epistemic policies and practices, produces a kind of inconstancy in doxastic commitments. This is a large and important subject, but here let us set it aside, in order to better attend to other kinds of inconstancy that might exist without, or independently of, inconstancy driven by learning, or by shifting bodies of evidence. For a similar reason, let us also set aside changes arising from forgetting. Belief changes that occur through learning and forgetting, then, are not in themselves indications of stake-sensitivity.

The sensitivity of a stake-sensitive belief has to do with its strength. That is, the strength with which it is held, the strength of the believer's doxastic commitment to it. Other properties of beliefs, such as their evidential support or their justification, also have strengths, but for present purposes, set those aside. What 'strength of doxastic commitment' amounts to is open to further interpretation, but whether a belief's strength is stake-sensitive seems a question that can be raised, whatever the precise interpretation. In particular, it can be raised whether belief is conceived as coming in a continuous range of strengths (degrees of belief), or conceived as falling into some specified set of categories of strengths, such as belief,

${ }^{3}$ To give an example, here is one possible avenue toward a treatment of stake-sensitive belief: Suppose degrees of belief are indicated by the odds at which the believer will accept bets, and suppose further that one's acceptable odds for large bets on $p$ differ from the acceptable odds for small ones. A theorist might regard this as reason for thinking the strength of the belief that $p$ is indefinite and stake-sensitive. In a later section we will see an account along those lines. In this section we will notice other possible explanations. But the issue is a general one, not confined to any particular strength-of-belief account. 
disbelief, withholding, doubt, moral certainty, and so on (call these categorical beliefs). Whatever framework we use for strengths, might the strength of my opinion about the locale of the toadstool example depend on the importance I attach to it?

The importance of the belief is what you take to be the significance of its truth or falsehood. Sometimes you may also attach significance to being in a state of believing it, as Pascal suggested, for example, in his wager. Being in a particular belief state may have causal (or supernatural!) consequences that matter to you, and those expected consequences may provide an incentive that in one way or another influences your belief. However viable that scenario is, here I set it aside. When I ask whether beliefs are stake-sensitive, I ask whether their strengths depend upon what is at stake on their truth, not on what is at stake on believing them.

Notice that the question is whether beliefs are (sometimes) stake-sensitive, rather than whether rational beliefs are. The latter question is also interesting, of course, but here I do not narrow the question by considering only beliefs that satisfy some significant norm(s) of rationality. In particular, I do not mean to focus only on beliefs that are justified, or on beliefs that are knowledge.

Think of stake-sensitivity as a specific kind of context-sensitivity. Your present expectations about the significance of $p$ 's being true depend on elements of your present context. But those expectations may influence what you think and do in many ways. In the interest of narrowing our attention to possible influence on the strength of your belief that $p$, let us notice other things the stakes may influence: Your expectations about what is at stake on $p$ will often influence where you direct your attention, and what you are disposed to say, and how cautiously you make decisions. And the importance of $p$ is widely thought to influence how well your belief that $p$ meets standards of justification and knowledge. So when the radio station calls and offers a good prize for your answer, the stakes may focus your attention more than the student's question did, and they may bring about shifts in the relevant standards for your being justified, or knowing. If you must bid for a chance to answer, you may become cautious when the bidding gets high. It might turn out that one or more of these influences arise through an influence on the strength of your belief (weakened belief at some point precludes knowledge, for example). But it is entirely possible that stakes influence these other things more directly, via other pathways, and without influencing the strength of your belief. We should be careful, then, not to mistake these other stakesensitivities for stake-sensitive states of belief.

So if stake-sensitivity of belief differs from these other sensitivities, what is it? A characteristic of a synchronic belief state that yields, in the presence of one set of stakes, belief of one strength, and in the presence of different stakes, belief of another strength. A stake-sensitive belief would be a mixed state of a certain sort, a state that resolves into beliefs having particular strengths depending upon the contextual stakes. If we think of belief strengths as degrees of doxastic influence in deliberation and choice represented by betting quotients, for example, stake-sensitive beliefs exhibit indeterminacy in the betting quotients, depending upon the stakes. If we think of belief strengths as estimates of expected truth 
value, stake-sensitive beliefs exhibit indeterminacy in those estimates. Call beliefs that are not stake-sensitive in this way stake-invariant beliefs.

\section{Stake-sensitive belief?}

I have already alluded to the difference between accounts of categorical strengths of belief, and accounts of degrees of belief. The difference between beliefs modeled in those two ways has often been discussed, usually with a focus on the relationship between degrees of belief and some conception of wholehearted full belief. It is not always clear, however, that what is under discussion (on the categorical side) is everyday belief, or instead a more complex notion, better labeled, as it often is, acceptance. There are many accounts of acceptance; typically they regard it as a state that answers to evidence and to something else - often a pragmatic something else-as well. Theories of acceptance, then, may be a promising place to look for sensitivity to influences by pragmatically significant stakes. But when such accounts allow judgments that one accepts $p$, but does not believe it, or vice versa, they are something other than accounts of stake-sensitive belief. Further, we often find them focused on rational acceptance, and they often exclude stake-sensitivity as irrational.

Along these lines, Kyburg $(1988,146-47)$ discusses the connection between rational credence and full belief (acceptance), and explicitly rules out stake-sensitive (rational) full belief. Christensen $(2004,28-29)$ briefly entertains the possibility of stake-sensitive dogmatic belief, but expresses no judgment. Nozick (1993, 96-97) makes the rationality of full belief a decision-theoretic matter, and highly context-dependent. He presents an example that suggests stake-sensitivity, though it appears likely, given his treatment, that the stakes attach to the act of believing rather than to the truth of the belief.

Work on the context-sensitivity, and the stake-sensitivity, of justification and knowledge sometimes raises the possibility of stake-sensitive belief. Fantl and McGrath (2002) offer an account of stake-sensitive justification, and along the way appear to consider whether belief, or at least rational belief, is stake-sensitive; it is not an issue they focus on, but they seem to think not.

Brian Weatherson (2005) brings us considerably closer to what I have in mind. He develops a 'probability first' account that encompasses degrees of belief and categorical belief. Weatherson advances the view that pragmatics matter not to justification, but to rational categorical belief. The paper is offered as an exploration of a possible account; in it, categorical belief is treated as a psychological phenomenon grounded on an underlying system of degrees of belief, a phenomenon that serves us in practical reasoning. Rational degrees of belief are responsive to evidence rather than to pragmatic influences, but categorical belief in $p$ is associated with having thoroughly incorporated $p$ into the basis of one's preferences for action. This will be influenced by one's degree of belief that $p$, and also by how the values of live options among which one can choose depend on $p$. 
"In cases like [Fantl and McGrath's], interests matter not because they affect the degree of confidence that an agent can reasonably have in a proposition's truth. (That is, not because they matter to epistemology.) Rather, interests matter because they affect whether those reasonable degrees of confidence amount to belief. (That is, because they matter to philosophy of mind.) There is no reason here to let pragmatic concerns into epistemology." (Weatherson 2005, 435-36)

Note the idea that degrees of belief are stake-invariant. The specific development of the view that categorical beliefs are stake-sensitive is not a traditional treatment of categorical belief (which is no argument against it), but it is akin to some accounts of acceptance. The idea that stake-sensitivity lies outside epistemology is more familiar.

Looking in other directions, we can find more. Richmond Thomason, in a series of papers discussing human and artificial reasoners, has sketched a complex treatment of belief, interesting in many ways, and interesting for our purposes in that it envisions belief-states with contextual sensitivities that include stake-sensitivity. And the economist Robert Nau (1992) has developed a formal decision-theoretic model of imprecise degrees of belief that exhibit stake-sensitivity.

\section{Constructing Belief for the Occasion.}

Work on artificial reasoning drives home the fact that the needs of an effective reasoner or agent go well beyond just possessing a large system of passively stored, wellformed beliefs. The reasoner must also solve the serious and general problem of bringing appropriate, relevant beliefs to bear on the occasion at hand. Responsiveness and sensitivity to context is desirable, and some structure and mechanism enabling it is computationally mandatory. The structure and mechanism will operate on beliefs already well formed, but they will also include ways of generating well-formed beliefs in response to background doxastic states and the requirements at hand. Thomason's framework for reasoners and their beliefs includes both an element of belief-generation, and an element of modularity that provides sensitivity to context:

"Rather than appealing to a global, monolithic attitude, we construct belief-like attitudes for the occasion at hand out of a large stock of potential beliefs that can be combined much as we might select and combine propositional axioms for some ad hoc purpose. ... When we combine potential beliefs into a modality that will guide our actions in a given situation, we can manipulate the beliefs by filtering out less plausible proto-beliefs in the presence of risk, or allowing them in when it is urgent to have a belief of some sort." (Thomason 2007, 476, my emphasis) 
The urgency to have a belief often derives from a need to act. Thomason (2009) presents a qualitative belief-intention-desire framework. Intentions require beliefs, and in order to come to the point of forming an intention, we may feel pressure to arrive at a belief:

In fact, however, human beliefs are influenced by a sense of risk. Without any change in the available evidence, a belief can disappear in the presence of risk, and can appear in the absence of risk.

... This mechanism of adjusting beliefs to risk would not be possible with monolithic belief-in the absence of new information, there would be no adjustment to be made. But if beliefs are ad hoc, and if one criterion for choosing the beliefs that are appropriate for a reasoning situation is a qualitative measure of the expected utility of acting on them, we can begin to explain how such adjustments can occur... (Thomason 2009, section 5)

The qualitative belief-intention-desire framework used here is compatible with using the minimal categorical set, believe, disbelieve, withhold. A new contextually appropriate belief generated from a dispositional proto-belief is not quite the same as a belief with a newly definite strength, contextually resolved from a dispositional mixed doxastic state. But it may not be far off, depending in part on how proto-beliefs are understood. In any case, it is quite clear that Thomason takes pragmatic factors, including the degree of risk, the significance of what is at stake, to be influences on the extent to which we hold various beliefs. Thomason's approach is noteworthy for its potential to yield rich, complex, and perhaps more satisfactory models of our doxastic states. It is also noteworthy in its departure from accounts of belief much more commonly used in epistemology and other philosophical work.

\section{Imprecise Belief-strengths and Stake-sensitivity.}

If beliefs are stake-sensitive, their strengths are indefinite until contextual stakes are supplied. The beliefs are persistent doxastic states, yet they exert degrees of influence on choice and inference that vary in the presence of different stakes on their truth. This indefiniteness might be seen as a kind of imprecision, and that suggests that we look for stakesensitivity in treatments of imprecise strengths of belief; such treatments can be found in the extensive literature on imprecise probability.

Imprecise beliefs are sometimes understood as states that have precise strengths, though they cannot be precisely measured, and sometimes as belief states that simply lack precise strengths (Walley 1991, Levi 1985). Call these imprecisely measured beliefs, and indeterminate beliefs, respectively. Our present interest is in the latter, in beliefs whose strengths are to some extent indeterminate. Imprecise beliefs are sometimes represented with ranges of values, intervals perhaps. But a better way uses sets of credence functions to 
represent states of imprecise belief. A function in the set that represents your beliefs attributes strengths to each of your beliefs, including a strength to your belief in $p$; the set of all the strengths in $p$ attributed by all your functions typically contains a range of values having unequal upper and lower bounds. The upper and lower bounds of your belief that $p$ are important characteristics of it, but your set of credence functions carries more information than that, about the relations between your belief that $p$ and your other beliefs. In treatments of rational imprecise beliefs, the credence functions are often probability functions, though not always standard ones.

Beliefs guide deliberation. Preferences and choices provide evidence about belief; we can better understand which beliefs are present by understanding the preferences and choices to which they are guides. Imprecise beliefs guide deliberation, but they speak less clearly. This means both that decision-making is less constrained, and that preferences and choices indicate beliefs less clearly. If stake-sensitivity is added to the imprecision that comes from lack of information and cognitive limitations, we add a further kind of complexity. To establish a stronger linkage between beliefs and choices we need information or principles about how sets of credence functions exert a refined influence on deliberation (through sharpening, filtering, or dominance, for example), and about how choices are evaluated (expected utility, non-expected utility, etc.)

Is stake-sensitivity a source of indeterminacy that motivates treatments of imprecise belief? Accounts of (indeterminate) imprecision typically regard it as arising because a) the believer lacks information, or has inconsistent information, about $p$, or b) the believer has cognitive limitations, or both. Peter Walley offers a survey of motivations for theories of imprecise belief; he lists fourteen sources of imprecision (1991, 212-17) and eleven arguments for taking it seriously (1991, 3-6). Many of them apply to imprecisely measured beliefs, but all of those that apply to indeterminate beliefs involve either (a) or (b). Those sources of imprecision are not incompatible with stake-sensitivity, but they provide no particular reason for regarding belief as sensitive to what's at stake. So we should not be surprised to find that treatments of imprecise belief and imprecise probability do not often address stake-sensitive belief. But there is at least one such treatment that does.

\section{Two views, Pro and Con.}

So far we have raised the question of whether beliefs as stake-sensitive, but not said much about the patterns sensitivity might produce - for examples, that high stakes tend to yield belief that is a) weaker, or anyway b) less precise. Scenarios that seem relevant, such as the one we began with about the radio quiz game, may fit such patterns, but perhaps others do not. In a given context, a less precise (strength of) belief is a vaguer commitment by the believer than a more precise belief would be. Vaguer belief-strengths can contribute to indecisiveness, but when a belief is imprecise without being completely so, it may nevertheless give clear guidance to the pursuit or avoidance of some available option, if the option is sufficiently good or bad. If high stakes produced the vagueness, the high-stakes decision suggests that the decision-maker has second-order confidence in the strength of the 
(admittedly imprecise) belief. This idea is developed by Robert Nau (1992) in an account of rational imprecise beliefs, an account that explicitly models stake-sensitive belief. ${ }^{4}$

Nau takes imprecise beliefs to be subject to dilation or narrowing when the contextual stakes are high or low. The general pattern in Nau's theory is that higher stakes render beliefs less precise, and that the higher stakes at which one would be guided by those beliefs indicate one's greater confidence in them. The account is an axiomatic theory of rational preferences over lotteries, from which degrees of belief (probabilities) and utilities can be found. It is a theory of imprecise belief; the belief (probability) functions associated with a given set of preferences yield a range of values for $\operatorname{prob}(p)$, with upper and lower bounds indicating the extent of imprecision. The theory employs weaker-than-usual axioms of completeness and transitivity that have the effect of guaranteeing that: 1) Preferences can be diluted but not necessarily 'undiluted;' when stakes are diminished, preferences are preserved, but when stakes are enhanced, maybe not. 2) Chains of preferences among lotteries are only guaranteed to be transitive when the stakes are diminished in proportion to the length of the chains; otherwise preferences may exhibit intransitivities.

Nau measures stakes by potential loss; the strength of a belief that $p$ is sensitive to the potential loss from a bet on or against $p$. The imprecise $\operatorname{prob}(p)$ is not fixed; its range expands and contracts as stakes are enhanced or diminished. Imagine, for example, that for a ticket on $p$ that pays off at even odds (for a betting quotient of .5) I would pay $\$ 20$, and for \$20 I would sell a ticket on $p$ that pays off at 2:3 (for a betting quotient of .6). For $\$ 100$ I would want a ticket that pays off at $3: 2(b q=.4)$, and I would offer for $\$ 100$ a ticket that pays off at 1:3 (bq $=.75)$. Then my $\operatorname{prob}_{20}(p)=[.5, .6]$, and my more imprecise $\operatorname{prob}_{100}(p)=[.4, .75]$. Parameters that capture the dilation and contraction of the bounds are confidence weights attached to the beliefs. Nau associates ratios of stakes with ratios of confidence, and would be willing to say, for example, that I have fivefold confidence in the more imprecise interval [.4, $.75]$, compared to my confidence in $[.5, .6]$. More generally, my imprecise prob(p) is associated with a sequence of nested intervals and corresponding confidence weights. Nau suggests that we think of the sequence of intervals as a single, fuzzy-edged probability interval.

Nau's theory is normative and so it imposes regularities that less-than-fully-rational beliefs violate, whether they are stake-sensitive or not. We can extract from it the underlying less-normative idea that imprecise degrees of belief may vary in their imprecision, and may shift in location, out of sensitivity to what is at stake. In general, whether we take that idea seriously partly depends on how fruitfully it can be developed; Nau's theory is an actual fruit well worth considering.

Let us turn to another view. Jim Joyce (2010, 311-313) discusses, among other topics, the guidance that imprecise rational beliefs provide to rational choice. When our beliefs are imprecise, arriving at a decision involves editing them to reduce the imprecision through sharpening. But, in contrast to Nau's theory, Joyce does not think the sharpening happens to the belief state; instead, it is a pragmatic component of deliberation.

\footnotetext{
${ }^{4}$ Thanks to Teddy Seidenfeld for bringing it to my attention.
} 
Think of credence functions in the set that characterizes your imprecise beliefs as members of your credal committee. Sharpening involves the deletion of members of your credal committee, and in principle there are many ways to do it. It might be done symmetrically (i.e. throw out equally distant members on either side of the midpoint value), or not. Joyce focuses on rational belief, and his view is that epistemic rationality offers no guidance to the (rational) refinement of imprecise belief during deliberation. If the evidence for $p$, however incomplete it may be, is the only legitimate source of epistemic justification for a particular way of sharpening, then there is no reason to expect an epistemic justification for any general policy about how to do it, including doing it symmetrically. Sharpening may be reasonable, but not on epistemic grounds.

Epistemically speaking, it is a matter of breaking ties. Pragmatically speaking, maybe not. Context, including what is at stake, influences how much sharpening we need to arrive at a decision. To explore this in detail we need to consider what constitutes a basis for a clear choice (must all committee members endorse it?), and what patterns of sharpening are at work (are the results smooth? unimodal? symmetric?). Some methods of progressive sharpening lead to points of clear decision that that further sharpening will not reverse. If decisions require committees to be unanimous, then higher stakes tend to demand more sharpening. One reason for that, I would say, is that compared with low stakes decisions, differences among high-stakes payoffs may have real significance even when they are smaller proportions of what is at stake. So greater guidance from belief is needed to make discernments among them. But, according to Joyce, this is all a matter of pragmatics for generating pseudo-belief; the imprecise belief is unchanged, and not stake-sensitive.

Joyce's view of imprecise belief pretty strictly associates imprecision with the absence or paucity of the believer's evidence. When evidence is lacking, imprecision is appropriate, and policies for reducing imprecision that have no basis in evidence lack epistemic justification. The rational believer will not alter his imprecise beliefs in such ways, and nothing in Joyce's discussion suggests that our doxastic states should be stake-sensitive. On the other hand, Joyce almost exclusively discusses rational belief and choice, and believers do not always believe what they should. As a psychological matter, Joyce allows, belief-states themselves are sometimes sharpened. If stakes influence the belief-state sharpening (something Joyce does not explicitly consider), those beliefs are stake-sensitive.

Joyce and Nau differ on whether rational imprecise belief is stake-sensitive. In Nau's theory, beliefs are more imprecise - the credal committee is enlarged - when stakes are high; this means that the set of high-stakes choices for which the committees give clear recommendations is reduced. Joyce asks what we are to do when imprecise beliefs give unclear recommendations, and describes a nondoxastic aspect of deliberation that generates more clarity. He further suggests that deliberative sharpening tends to be more pronounced when stakes are high, which appears to fit Nau's idea that high stakes dilate imprecise beliefs. But since Joyce regards precision and imprecision as responses to evidence, he is unlikely to endorse the view that rational beliefs are more or less precise, or otherwise affected, by what is at stake. If more sharpening is often needed when stakes are high, that has more to do with the level of doxastic guidance needed to discriminate among options with high-stakes 
consequences. A more fundamental difference is this: Nau's theory identifies degrees of imprecise belief with their influences on deliberation, in a way that is common to many preference-based accounts of belief. Joyce sees (rational) degrees of imprecise belief as purely doxastic responses to evidence; they guide deliberation through surrogates that are not, strictly speaking, what the decision-maker believes.

\section{Conclusion.}

We have considered whether beliefs are stake-sensitive, or whether it would be enlightening to so treat them in our theories. Relatively few accounts do that, and models of imprecise belief are typically not directed toward the idea. Whether the infrequent attention reflects implicit rejection of the idea, or just neglect, is difficult to say. But treatments such as Thomason's and Nau's and Weatherson's, and their motivations for providing them, suggest that the idea is worth more consideration.

A further point is that, since accounts of stake-sensitive belief are infrequent in epistemology, subjective degree-of-belief (probability) theories that follow the trend are not exceptional in doing so. This is worth noticing, because such theories of action-guiding belief often attract the criticism that they attend to states that are not sufficiently doxastic, states that are inappropriate for epistemology. I disagree with the criticism, but that is a topic for another occasion. Here I would say that, while the cogency of the criticism is not entirely decided when we settle on a view concerning the stake-sensitivity or stake-invariance of belief, attention to that issue helps to clarify the dispute. 


\section{References}

Armendt, Brad. 2010. "Stakes and Beliefs." Philosophical Studies 147: 71-87.

Christensen, David. 2004. Putting Logic in its Place. Oxford: Clarendon Press.

Fantl, Jeremy, and Matthew McGrath. 2002. "Evidence, Pragmatics, and Justification." Philosophical Review 111: 67-94.

Joyce, James M. 2010. “A Defense of Imprecise Credences in Inference and Decision Making." Philosophical Perspectives 24: 281-323.

Kyburg, Henry. 1988. "Full Belief.” Theory and Decision 25:137-162.

Levi, Isaac. 1985. "Imprecision and Indeterminacy in Probability Judgment." Philosophy of Science 52: 390-409.

Nau, Robert. 1992. "Indeterminate Probabilities on Finite Sets." Annals of Statistics 20:17371767.

Nozick, Robert. 1993. The Nature of Rationality. Princeton: Princeton University Press.

Thomason, Richmond. 2007. "Three Interactions Between Context and Epistemic Locutions." In Modeling and Using Context, ed. Boicho N. Kokinov, Daniel C. Richardson, Thomas Roth-Berghofer and Laure Vieu, 467-481. Berlin: Springer.

Thomason, Richmond. 2009. "Belief, Intention, and Practicality: Loosening up Agents and their Propositional Attitudes."

Walley, Peter. 1991. Statistical Reasoning with Imprecise Probabilities. London: Chapman and Hall.

Weatherson, Brian. 2005. “Can We Do Without Pragmatic Encroachment?” Philosophical Perspectives 19: 417-443. 